[3] F. Hausdorff, Mengenlehre, Berlin 1935.

[4] H. Johnen, Inequalities connected with the moduli of smoothness, Mat. Vestnik 9 (1972), pp. 289-303.

[5] D. Leviatan, The rate of approximation in the $I_{1^{-n} \text { orm }}$ by some Bernstein-type operators (unpublished manuscript).

[6] G. G. Lorentz, Bernstein polynomials, Univ. of Toronto Press, Toronto 1953.

[7] A. Lupass, Some properties of the linear positive operators (I), Mathematica (Cluj) 9 (32) (1967), pp. 77-83.

[8] A. Lupaş and M. W. Müller, Approximation properties of the $M_{n}$-operators, Aequationes Math. 5 (1970), pp. 19-37.

[9] W. Meyer-König and K. Zeller, Bernsteinsche Potenzreihen, Studia Math. 19 (1960), pp. 89-94.

[10] M. W. Müller, Gleichmäßige Approximation durch die Folge der ersten Ableitungen der Operatoren von Meyer-Kömig und Zeller, Math. Z. 106 (1968), pp. 402-406.

[11] - Degree of $L_{p}$-approximation by integral Schoenberg splines, J. Approximation Theory 21 (1977), pp. 385-393.

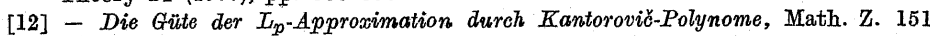
(1976), pp. 243-247.

[13] W. Orlicz, Ein Satz über die Erweiterung von linearen Operationen. Studia Math. 5 (1934), pp. 127-140.

[14] J.Peetre, A theory of interpolation of normed spaces, Lecture Notes, Brazilia 1963.

[15] P. C. Sikkema, On the asymptotic approximation with operators of Meyer-König and Zeller, Indag. Math. 32 (1970), pp. 428-440.

[16] A. Zygmund, Trigonometric series, Vol. I. and II, Cambridge University Press, London-New York 1968.

\section{Some characterizations of the $n$-dimensional Peano derivative}

by

ISRAEL BERNARD ZIBMAN (Princeton, N.J.)

Abstract. A measurable function $f$ is said to have a Peano derivative of order te at a point $x$ if there is a polynomial $P$ of degree at most $k$ with the property that $f(x+t)=P(t)+o(|t|)^{k}$. This work gives a characterization of the Peano derivative for functions of several variables in terms of the behaviour of the expression

$$
\sum_{i=1}^{N} A_{i} f\left(x+\varrho \sigma\left(v_{i}\right)\right)-\left(\sum_{i=1}^{N} A_{i}\right) f(x) .
$$

The $A_{i}$ are real numbers and the $v_{i}$ are points on the unit sphere, $\varrho>0$ and $\sigma \in \mathrm{S} O(n)$. Almos $A_{i}$ are realts similar to those of Denjoy-Rademacher-Stepanov, KhinAlmost everywhere results similar to those of Denjoy-Rade are obtained in this non-linear setting.

The techniques involve boundary behavior of harmonic functions and analysis on $S O(n)$. When $n$ is greater than 2 the non-commutativity of $\mathrm{SO}(n)$ requires special on one to substitute a certain convolution with a central function for a convolution with ane to substitute

Introduction. The purpose of this paper is to present an extension and a unification of several of the characterizations of the $n$-dimensional Peano derivative. Our characterizations will be stated as a description Peano derivative. Of functions restricted to spheres centered at points of possible differentiability. The action of the rotation group on the sphere will play a significant role.

We say that a function $f$, defined on a neighborhood of a point $x$ in $\boldsymbol{R}^{n}$, has a kth Peano derivative at $x$ if there is a polynomial $P$ of degree at most 7 such that $f(x+t)=P(t)+o\left(|t|^{k}\right)$. When $k=1$, this is the ordinary derivative. When $k$ is greater than $1, f$ need not be $k-1$ differentiable near $x$ to have a $k$ th Peano derivative at $x$.

We consider in this paper configurations consisting of a finite number of points on the unit sphere in $\boldsymbol{R}^{n}, v_{1}, \ldots, v_{N}$. We assign each point a non-zero weight $A_{i}$. The origin is given the weight $B=-\sum_{i=1}^{N} A_{i}$. To each configuration we associate an integer type $m$. The integer $m$ is defined as the infimum of the degrees of all polynomials for which $\sum_{i=1}^{N} A_{i} P\left(v_{i}\right)+$ 
$+B P(0) \neq 0$. Since $N$ is finite, $m$ is also finite and non-zero. The type is a measure of the symmetry - all moments of order less than $m$ vanish.

We shall consider the question of when the condition

$$
\sum_{i=1}^{N} A_{i} f\left(x+\varrho \sigma\left(v_{i}\right)\right)+B f(x)=O\left(\varrho^{k}\right) \quad(\varrho>0, \sigma \in \mathrm{SO}(n))
$$

for $x$ in a measurable set $E$ implies that $f$ has a $k$ th Peano derivative almost everywhere in $E$. We prove that this is true when $k$ equals $m$. When $k$ is less than $m$, an integral similar to an integral of Marcinkiewicz must be finite. We recall the following classical theorems to illustrate the situation. In each case, $f$ is a measurable function defined on a neighborhood of a measurable subset $E$ of $\boldsymbol{R}^{n}$. In the literature, the theorems of Khintchine and Marcinkiewicz-Zygmund are stated for functions of one variable. The extension to $n$ variables is an easy application of the methods of Stein and Zygmund ([10], Chapter VIII). The theorems below are also contained in the present paper.

Denjoy-Rademacher-Stepanov [7]: $f$ has an ordinary first derivative almost everywhere in $E$ if and only if $f(x+t)-f(x)=O(|t|)$ for almost every $x \in E$.

Khintchine [3]: $f$ has an ordinary first derivative almost everywhere in $E$ if and only if $f(x+t)-f(x-t)=O(|t|)$ for almost every $x \in E$. Stein-Zygmund [13]: $f$ has an ordinary first derivative almost everywhere in $E$ if and only if the following two conditions are satisfied for a.e. $x \in E$.

(1) $f(x+t)+f(x-t)-2 f(x)=O(|t|)$;

(2) There exists $\delta_{x}>0$ such that

$$
\int_{|t|<\delta_{x}} \frac{|f(x+t)+f(x-t)-2 f(x)|^{2}}{|t|^{n+2}} d t<\infty .
$$

Marcinkiewicz-Zygmund [6]: $f$ has a second Peano derivative almost everywhere in $E$ if and only if $f(x+t)+f(x-t)-2 f(x)=O\left(|t|^{2}\right)$ for a.e. $x \in E$.

Stein-Zygmund [8]: Let $v_{i}=(0,0, \ldots, 1,0, \ldots, 0), i=1, \ldots, n, b e$ the standard basis for $\boldsymbol{R}^{n}$. f has a second Peano derivative almost everywhere in $E$ if and only if $\sum_{i=1}^{n}\left(f\left(x+\varrho \sigma\left(v_{i}\right)\right)+f\left(x-\varrho \sigma\left(v_{i}\right)\right)\right)-2 n f(x)=O\left(\varrho^{2}\right)$ for a.e. $x \in E$. The estimate may depend on $x \in E$ but not on $\sigma \in \mathrm{SO}(n)$.

Observe in the above theorem that if $f$ is twice differentiable at $x$, then $\varrho^{-2} \cdot\left(\sum_{i=1}^{n}\left(f\left(x+\varrho \sigma\left(v_{i}\right)\right)+f\left(x-\varrho \sigma\left(v_{i}\right)\right)\right)-2 n f(x)\right)$ converges to the Laplacian of $f$ at $x$. The above theorem may be viewed as a local analog of the following theorem.
Theorein ([10], p. 77). Let $(\partial / \partial x)^{a}$ be a differential monomial of degree 2. If $f$ is continuously twice differentiable and has compact support, then

$$
\left\|\left(\frac{\partial}{\partial x}\right)^{\alpha} f\right\|_{L_{p}} \leqslant A_{p}\|\Delta f\|_{L_{p}} \text { for } .1<p<\infty .
$$

The last theorem has been generalized and extended to other elliptic systems [1]. The following version is very closely related to the results in this paper.

THEOREM. Let $P_{1}(x), \ldots, P_{d}(x)$ and $P(x)$ be homogeneous polynomials of degree $m$. Suppose the only common complex zeroes ${ }^{1}$ ) of $P_{1}, \ldots, P_{d}$ all lie on the variety $x_{1}^{2}+\ldots+x_{n}^{2}=0$. For $1<p<\infty$, there is a constant $A_{p}$ such that for all $f \in C^{m}\left(\boldsymbol{R}^{n}\right)$ with compact support,

$$
\|P(\partial \mid \partial x) f\|_{L_{p}} \leqslant A_{p} \sum_{i=1}^{d}\left\|P_{i}(\partial / \partial x) f\right\|_{L_{p}} .
$$

The proof of this theorem is based on an easy application of the Hilbert Nullstellensatz and the boundedness of the higher Riesz transforms for $1<p<\infty$.

The proof of the theorems in this paper will use the local analog of the Riesz transform theory - the theory of nontangential limits for conjugate systems of harmonic functions. The $P_{1}, \ldots, P_{d}$ will be homogeneous harmonic polynomials. These polynomials transform suitably under the action of the rotation group. We let $u(x, y)$ be the Poisson integral of our function $f$. We shall gain control of $P_{i}(\partial / \partial x) u(x, y), i=1, \ldots, d$. The method of harmonic derivatives of Stein [10], [11] will be used to complete the proof.

The early theorems about differentiability were concerned with essentially linear configurations. In the theorems of Khintchine and Marcinkiewicz-Zygmund and the earlier theorem of Stein-Zygmund, the second point, $-t$, is in the opposite direction of the first point. When other directions are introduced, as in the second theorem of Stein-Zygmund, we are led in the proof to consider expressions of the following type.

$$
\sum_{i=1}^{N} \int_{0}^{\infty} \int_{\sigma \in \mathrm{SO}(n)} P\left(y, \varrho, r, \sigma\left(v_{i}\right) \cdot v_{i}\right) A_{i} f\left(x+\varrho \sigma\left(v_{i}\right)\right) d \sigma \varrho^{n-1} d \varrho .
$$

The function $P(y, \varrho, r, \sigma(v) \cdot v)$ will be some derivative of the Poisson kernel expressed in spherical coordinates. In two dimensions, $\sigma\left(v_{i}\right) \cdot v_{i}$ $=\sigma\left(v_{j}\right) \cdot v_{j}$ for any unit vectors $v_{i}, v_{j}$ and $\sigma \in \mathrm{SO}(2)$. We can then move

(1) This theorem is true under the weaker assumption that the only real zero is at the origin. The corresponding local analog is not yet known. (See [9], p. 166.) 
the summation sign inside the integral, past the function $P(y, \varrho, r, \sigma(v) \cdot v)$. In higher dimensions this is not possible since $\mathrm{SO}(n)$ is not commutative.

To overcome this problem we develop a technique introduced by Stein-Zygmund [8]. They demonstrated the existence of a function $\tilde{P}(y, \varrho, r, \sigma)$ which is a central function of $\sigma \in \mathrm{SO}(n)$ and satisfies

$\int_{\mathrm{SO}(n)} \tilde{P}(y, \varrho, r, \sigma) f(\sigma(v)) d \sigma=\int_{\mathrm{SO}(n)} \frac{\partial^{2}}{\partial y^{2}}\left(\frac{c_{n} y}{\left(\varrho^{2}+r^{2}-2 \varrho r \sigma(v) \cdot v+y^{2}\right)^{\frac{1}{2}(n+1)}}\right) f(\sigma(v)) d \sigma$

for any $v$ on the unit sphere. It is also shown that $\tilde{P}$ is comparable in size to the indicated second derivative of the Poisson kernel. The proof uses the theory of semigroups and Schauder type estimates. The proof in this paper is more direct and extends to other derivatives of the Poisson kernel. This will be carried out in Section II.

I would like to express my appreciation to my adviser, E. M. Stein who suggested the problem and who provided much advice and encouragement during the course of this work.

I. The characterizations. Let $v_{1}, \ldots, v_{N}$ be a finite collection of points on the unit sphere in $\boldsymbol{R}^{n}$. Let $A_{1}, \ldots, A_{N}$ be non-zero real numbers. Set $B=-\sum_{i=1}^{N} A_{i}$. Let $m$ be the infimum of the degrees of all polynomials for which $\sum_{i=1}^{N} A_{i} Q\left(v_{i}\right)+B Q(0) \neq 0$. We note that $m$ is greater than zero. Let $f$ be a measurable function defined on a neighborhood of a measurable subset $E$ of $\boldsymbol{R}^{n}$. We obtain the following theorems.

THEOREM 1. The function $f$ has an $m$-th Peano derivative at almost every $x \in E$ if and only if for $\varrho>0, \sigma \in \mathrm{SO}(n)$,

$$
\sum_{i=1}^{N} A_{i} f\left(x+\varrho \sigma\left(v_{i}\right)\right)+B f(x)=O\left(\varrho^{m}\right) \quad \text { as } \quad \varrho \rightarrow 0 \quad \text { for a.e. } x \in E .
$$

The estimate may depend on $x$ but is uniform in $\sigma$ for fixed $x$.

THeOReM 2. If $k<m$, then $f$ has a k-th Peano derivative at almost every $x \in E$ if and only if for a.e. $x \in E$ the following two conditions are satisfied.

(a) $\sum_{i=1}^{N} A_{i} f\left(x+\varrho \sigma\left(v_{i}\right)\right)+B f(x)=O\left(\varrho^{k}\right)$.

(b) There is a $\delta_{x}>0$ such that

$$
\int_{0}^{\delta_{x}} \int_{\mathrm{SO}(n)} \frac{\left|\sum_{i=1}^{N} A_{i} f\left(x+\varrho \sigma\left(v_{i}\right)\right)+B f(x)\right|^{2}}{\varrho^{1+2 k}} d \sigma d \varrho<\infty .
$$

The estimate in (a) is uniform in $\sigma$ for fixed $x$.
Observe that the conditions for differentiability in Theorem 2 are independent of the type of the configuration as long as $k$ is less than $m$. This might seem surprising, since, when $m$ is very large, the cancellations would cause $\sum_{i=1}^{N} A_{i} f\left(x+\varrho \sigma\left(v_{i}\right)\right)+B f(x)$ to be small for any reasonable function $f$. The method of Marcinkiewicz [5] shows that for any configuration and $\varepsilon>0$, one can find a function possessing an ordinary first derivative almost everywhere, yet

$$
\int_{0}^{\delta_{x}} \int_{\operatorname{so}(n)} \frac{\left|\sum_{i=1}^{N} A_{i} f\left(x+\varrho \sigma\left(v_{i}\right)\right)+B f(x)\right|^{2-\varepsilon}}{\varrho^{3-\varepsilon}} d \sigma d \varrho
$$

is infinite almost everywhere for any $\delta_{x}>0$.

The proof of Theorems 1 and 2 will be based on the idea of "splitting of functions" developed by Marcinkiewicz [4] and Calderón and Zygmund [1]. It will use the method of harmonic derivatives due to Stein and Zygmund [10], [13]. This method is described by the following definitions and theorem from Stein [10], Chapter VIII. In Stein's book the statements are for first derivatives, but the extension to higher derivatives is immediate.

DefrntTron. Let $f$ be a locally integrable function defined in an open set $\Omega$. For a fixed $x_{0} \in \Omega$ we modify $f$ by setting it to zero outside a bounded neighborhood of $x_{0}$. The function $f$ is now in $L_{1}\left(\boldsymbol{R}^{n}\right)$ and we may take its Poisson integral

$$
u(x, y)=P_{y} * f(x)=\int_{\boldsymbol{R}^{n}} \frac{c_{n} y}{\left(|x-t|^{2}+y^{2}\right)^{\frac{1}{2}(n+1)}} f(t) d t .
$$

We shall say that $f$ has a $k$-th harmonic derivative at $x_{0}$ if for all nonnegative $n$-tuples $\alpha=\left(\alpha_{1}, \ldots, \alpha_{n}\right)$ with $\sum_{i=1}^{n} \alpha_{i} \leqslant k,(\partial / \partial x)^{\alpha} u(x, y)$ has a non-tangential limit at $x_{0}$. This means that there is a $\beta>0$ such that

$$
\lim _{\substack{\left|x-x_{0}\right|<\beta y \\ y \rightarrow 0}}\left(\frac{\partial}{\partial x}\right)^{a} u(x, y) \text { exists and is finite. }
$$

Definimion ([1]). A function $f \in L_{p}\left(\boldsymbol{R}^{n}\right), 1 \leqslant p \leqslant \infty$, has a $k$-th derivative in the $L_{p}$ sense at $x_{0}$ if there is a polynomial $Q$ of degree at most ${ }_{k}$ with the property that

$$
\left(\varrho^{-n} \int_{|t|<\varrho}|f(x+t)-Q(t)|^{p} d t\right)^{1 / p}=o\left(\varrho^{k}\right) \quad \text { as } \quad \varrho \rightarrow 0 .
$$

If $f$ has a $k$ th Peano derivative at $x_{0}$ or a $k$ th derivative in the $L_{p}$ sense, then $f$ has a kth harmonic derivative at $x_{0}$. Also if $f$ and all derivatives 
of order less than or equal to $k$ exist in the sense of distributions and are in $L_{\infty}$ (we write $f \in L_{\infty}^{k}$ ), then $f$ may be redefined on a set of measure zero to have a kth Peano derivative almost everywhere.

SPLITTANG THEOREM. Suppose that $f$ is a locally integrable function and that for every $x_{0}$ in a set $E$ of finite measure $f$ has a $k$-th harmonic derivative at $x_{0}$. Then for every $\varepsilon>0$ we can find a compact set $F$ with $m(E-F)<\varepsilon$ and a function $g \in L_{\infty}^{k}$ such that $b(x)=f(x)-g(x)$ vanishes on $F$. If, in addition, $f$ has a $k$-th derivative in the $L_{p}$ sense at every point of $E$, then we may choose $F$ as above with the additional property that

for $x_{0} \in F$.

$$
\int_{|y| \leqslant 1} \frac{\left|b\left(x_{0}+y\right)\right|^{p}}{|y|^{n+k p}} d y<\infty
$$

We proceed to prove Theorems 1 and 2

The necessity of the condition in Theorem 1 is clear. Suppose

$$
f(x+t)=\sum_{j=0}^{m} P_{j}(t)+o\left(|t|^{m}\right),
$$

where $P_{j}$ is homogeneous of degree $j$. Then

$$
\sum_{i=1}^{N} A_{i} f\left(x+\varrho \sigma\left(v_{i}\right)\right)+B f(x)=\sum_{j=1}^{m} \varrho^{j} \sum_{i=1}^{N} A_{i} P_{j}\left(\sigma\left(v_{i}\right)\right)+o\left(\varrho^{m}\right) .
$$

Since the configuration is of type $m, \sum_{i=1}^{N} A_{i} P_{j}\left(\sigma\left(v_{i}\right)\right)=0$ for $j<m$. Hence

$$
\sum_{i=1}^{N} A_{i} f\left(x+\varrho \sigma\left(v_{i}\right)\right)+B f(x)=\varrho^{m} \sum_{i=1}^{N} A_{i} P_{m}\left(\sigma\left(v_{i}\right)\right)+o\left(\varrho^{m}\right)=O\left(\varrho^{m}\right) .
$$

The necessity of condition (a) of Theorem 2 is proven in the same fashion. To show the necessity of condition (b), we first prove the following proposition.

Propostion. Let $\left\{A_{i}, v_{i}, i=1, \ldots, N\right\}$ be a configuration of type $m$. Let $k<m$. If $f \in L_{2}^{k}\left(\boldsymbol{R}^{n}\right)$, then

$$
\int_{\boldsymbol{R}^{n}} \int_{0}^{\infty} \int_{S_{0}(n)} \frac{\left|\sum_{i=1}^{N} A_{i} f\left(x+\varrho \sigma\left(v_{i}\right)\right)+B f(x)\right|^{2}}{\varrho^{1+2 k}} d \sigma d \varrho d x \leqslant C\|f\|_{L_{2}^{k}\left(\boldsymbol{R}^{n}\right)} .
$$

Proof. By Plancherel's theorem and the effect of translations on the Fourier transform, the left-hand side of (1) is equal to

$$
\int_{\mathrm{SO}(n) 0} \int_{0}^{\infty} \varrho^{-1-2 k} \int_{\boldsymbol{R}^{n}}|\hat{f}(t)|^{2}\left|\sum_{i=1}^{N} A_{i} e^{2 \pi i t \cdot \varrho \sigma\left(v_{i}\right)}+B\right|^{2} d t d \varrho d \sigma .
$$

Let

$$
I(t)=\int_{\mathrm{SO}(n)} \int_{0}^{\infty}\left|\sum_{i=1}^{N} A_{i} e^{2 \pi i t \cdot \varrho \sigma\left(v_{i}\right)}+B\right|^{2} \varrho^{-1-2 k} d \varrho d \sigma .
$$

For fixed $t$, Taylor's theorem shows that $\sum_{i=1}^{N} A_{i} e^{2 \pi i t \cdot \sigma \sigma\left(v_{i}\right)}+B=O\left(\varrho^{m}\right)$ as $\varrho \rightarrow 0$ so $I(t)$ is finite. By homogeneity, $I(t)=C|t|^{2 k}$. Hence (2) is equal to

$$
C \int_{\mathbf{R}^{n}}|t|^{2 k}|\hat{f}(t)|^{2} d t \leqslant C\|f\|_{L_{\mathbf{2}}^{k}\left(\mathbf{R}^{n}\right)} .
$$

If $f$ has a kth Peano derivative on $E$, then $f$ is locally bounded. We may restrict $E$ to a compact set and assume that $f$ vanishes outside a bounded neighborhood of $E$. Now the existence of a $k$ th Peano derivative implies that $f$ has a $k$ th derivative in the $L_{2}$ sense on $E$ and the Splitting theorem may be applied. We can find a set $F$, arbitrarily close to $E$ in measure and functions $g \in L_{\infty}^{k}\left(\boldsymbol{R}^{n}\right)$ and $b(x)=f(x)-g(x)$. The function $g$ is in $L_{2}^{k}\left(\boldsymbol{R}^{n}\right)$ and satisfies condition (b) of the theorem by the proposition. Since $b$ vanishes on $F$

$$
\begin{aligned}
\int_{\varrho \leqslant 1} \int_{\text {SO }(n)} \frac{\left|\sum_{i=1}^{N} A_{i} b\left(x+\varrho \sigma\left(v_{i}\right)\right)+B b(x)\right|^{2}}{\varrho^{1+2 k}} d \sigma d \varrho \\
\leqslant \sum_{i=1}^{N}\left|A_{i}\right| \int_{|y| \leqslant 1} \frac{|b(x+y)|^{2}}{|y|^{n+2 k}} d y<\infty \quad \text { for } \quad x \in F .
\end{aligned}
$$

Hence $f=g+b$ also satisfies condition (b).

The proof of the sufficiency of the conditions is more difficult. We will first show that it suffices to prove that $f$ has a harmonic derivative of the appropriate order. The following lemma will be used repeatedly for the purpose of "desymmetrization".

Destmmetrization Lemma. Let $E$ be a measurable subset of $\boldsymbol{R}^{n}$ of finite positive measure. $\chi_{E}$ will denote the characteristic function of $E$. Set $e=\frac{1}{2} \int_{0}^{1} \int_{\mathrm{SO}(n)} \varrho^{n-1} d \sigma d \varrho$. If $v_{1}, \ldots, v_{N}$ is a finite subset of the unit sphere in $\boldsymbol{R}^{n}$ and $0<\varepsilon \leqslant 1$, then for almost every $x_{0} \in E$ there is a $\delta_{x_{0}}>0$ such that if $\left|x-x_{0}\right|<\delta_{x_{0}}$, then

$\int_{\mathrm{SO}(n)} \int_{0}^{\varepsilon\left|x-x_{0}\right|} \chi_{E}\left(x-\varrho \sigma\left(v_{1}\right)\right) \prod_{i=2}^{N} \chi_{E}\left(x-\varrho \sigma\left(v_{1}\right)+\varrho \sigma\left(v_{i}\right)\right) \varrho^{n-1} d \varrho d \sigma>\sigma\left(\varepsilon\left|x-x_{0}\right|\right)^{n}$.

This says that if $x$ is close to $x_{0}$, there is a point of $E$ even closer to $x$ with the property that this point, $\left(x-\varrho \sigma\left(v_{1}\right)\right)$, is the center of a confi- 
guration containing $x=\left(x-\varrho \sigma\left(v_{1}\right)\right)+\varrho \sigma\left(v_{1}\right)$ in the $v_{1}$ position and such that the points $\left(x-\varrho \sigma\left(v_{1}\right)\right)+\varrho \sigma\left(v_{i}\right), i=2, \ldots, N$, in the $v_{i}$ position all belong to $E$. In fact, there are many such pairs $(\varrho, \sigma)$ with this property. In particular, there is some $r<\varepsilon\left|x-x_{0}\right|$ for which there are many $\sigma$. We have the following corollary.

CoRolLaRY. There is an $r<\varepsilon\left|x-x_{0}\right|$ with the property that

$$
\int_{\operatorname{so}(n)} \chi_{E}\left(x-r \sigma\left(v_{1}\right)\right) \prod_{i=2}^{N} \chi_{E}\left(x-r \sigma\left(v_{1}\right)+r \sigma\left(v_{i}\right)\right) d \sigma \geqslant n c .
$$

Proof of the corollary. If the integral was smaller than no for all $0<r<\varepsilon\left|x-x_{0}\right|$, then the integral of the lemma would be dominated by

$$
\int_{0}^{\varepsilon\left|x-x_{0}\right|} n c e^{n-1} d \varrho=c\left(\varepsilon\left|x-x_{0}\right|\right)^{n}
$$

Proof of the lemma. Let $\vec{\chi}=1-\chi_{E}$.

$$
\begin{aligned}
& \int_{\mathrm{SO}(n)} \int_{0}^{\varepsilon\left|x-x_{0}\right|} \chi_{E}\left(x-\varrho \sigma\left(v_{1}\right)\right) \prod_{i=2}^{N} \chi_{E}\left(x-\varrho \sigma\left(v_{1}\right)+\varrho \sigma\left(v_{i}\right)\right) \varrho^{n-1} d \varrho d \sigma \\
& =\int_{\mathrm{SO}(n)} \int_{0}^{e\left|x-x_{0}\right|} \varrho^{n-1} d \varrho d \sigma-\int_{\mathrm{SO}(n)} \int_{0}^{\varepsilon\left|x-x_{0}\right|} \bar{\chi}\left(x-\varrho \sigma\left(v_{1}\right)\right) \varrho^{n-1} d \varrho d \sigma- \\
& -\sum_{i=2}^{N} \int_{\mathrm{SO}(n)} \int_{0}^{\varepsilon\left|x-x_{0}\right|} \bar{x}\left(x-\varrho \sigma\left(v_{1}\right)+\varrho \sigma\left(v_{i}\right)\right) \varrho^{n-1} d \varrho d \sigma+ \\
& +\sum_{i=2}^{N} \int_{\mathrm{SO}(n)} \int_{0}^{\varepsilon\left|x-x_{0}\right|} \bar{x}\left(x-\varrho \sigma\left(v_{1}\right)\right) \bar{\chi}\left(x-\varrho \sigma\left(v_{1}\right)+\varrho \sigma\left(v_{i}\right)\right) \varrho^{n-1} d \varrho d \sigma+\ldots-\ldots
\end{aligned}
$$

The first integral after the equal sign is just $2 e\left(\varepsilon\left|x-x_{0}\right|\right)^{n}$. The remaining sum of integrals is dominated by

$$
2^{N+1} \int_{|t|<3\left|x-x_{0}\right|} \bar{x}\left(x_{0}+t\right) d t .
$$

When $x_{0}$ is a point of density of $E$, this integral is $o\left(\left|x-x_{0}\right|^{n}\right)$. We choose $\delta_{x_{0}}$ such that for $\left|x-x_{0}\right|<\delta_{x_{0}}$, this integral is less than $o\left(\varepsilon\left|x-x_{0}\right|\right)^{n}$.

Remarks. 1. The function $m_{x_{0}}(r)=m\left\{x \notin E,\left|x-x_{0}\right|<3 r\right\}$ is continuous. If we fix $\varepsilon>0$ and $\delta>0$, we see from the proof that the subset of $E$ for which the result of the lemma holds for $\delta_{x_{0}}=\delta$ is measurable. It is simply the set of $x_{0} \in E$ for which $2^{N+1} m_{x_{0}}(r)<c(\varepsilon r)^{n}$ for $r<\delta$.

2. If $E$ is not necessarily measurable it is still true that, except for a subset of Lebesgue measure zero, the points of $E$ are points of exterior density of $E$. (See $[10]$, p. 251.) The results of the lemma hold at these doints.
Now suppose that $f$ has a $k$ th harmonic derivative at every $x_{0} \in E$. We choose $F \subset E$ and write $f=g+b$ according to the splitting lemma. The function $g$ is in $L_{\infty}^{k}$ and has a $k$ th Peano derivative a.e. The function $b$ vanishes on $F$. To show that $b$ has a $k$ th Peano derivative on $F$, it will certainly suffice to show that $b\left(x_{0}+t\right)=o\left(|t|^{k}\right)$ for a.e. $x_{0} \in F$.

$$
\begin{aligned}
& \sum_{i=1}^{N} A_{i} b\left(x_{0}+\varrho \sigma\left(v_{i}\right)\right)+B b\left(x_{0}\right) \\
& \quad=\sum_{i=1}^{N} A_{i}\left(f\left(x_{0}+\varrho \sigma\left(v_{i}\right)\right)-g\left(x_{0}+\varrho \sigma\left(v_{i}\right)\right)\right)+B\left(f\left(x_{0}\right)-g\left(x_{0}\right)\right)=O\left(\varrho^{k}\right) .
\end{aligned}
$$

Consider the subset $F_{j}=\left\{x_{0} \in F:\left|\sum_{i=1}^{N} A_{i} b\left(x_{0}+\varrho \sigma\left(v_{i}\right)\right)+B b\left(x_{0}\right)\right|<j \varrho^{k}\right.$ for $\varrho<1 / j\}$. $F_{j}$ is given by a continuum of conditions and may not be measurable if $f$ is not continuous. Nevertheless, we may still apply the desymmetrization lemma to $F_{j}$ for any $\varepsilon, 0<\varepsilon \leqslant 1$. For a.e. $x_{0} \in F_{j}$, there is a $\delta_{x_{0}}>0$ with the property that for any $x$ with $\left|x-x_{0}\right|<\min \left(\delta_{x_{0}}, 1 / j\right)$ there is an $r, 0<r<\varepsilon\left|x-x_{0}\right|$, and $\sigma \in \mathrm{SO}(n)$ such that $x-r \sigma\left(v_{1}\right) \in F_{j}$ and $x-r \sigma\left(v_{1}\right)+r \sigma\left(v_{i}\right) \in F_{j}, i=2, \ldots, N$. The function $b$ vanishes at these points. Hence

$$
\begin{aligned}
\left|A_{1} b(x)\right| & =\mid \sum_{i=1}^{N} A_{i} b\left(x-r \sigma\left(v_{1}\right)+r \sigma\left(v_{i}\right)\right)+B b\left(x-r \sigma\left(v_{1}\right)\right) \\
& \leqslant j\left(\varepsilon\left|x-x_{0}\right|\right)^{k}=o\left(\left|x-x_{0}\right|^{k}\right) .
\end{aligned}
$$

Recall that $A_{1}$ is not zero. Since $F$ is equal to a countable union of the $F_{j}, b$ has a $k$ th Peano derivative almost everywhere in $F$ and hence in $E . f=g+b$, likewise, has a $k$ th Peano derivative in $E$.

The remainder of the paper will be devoted to showing that $f$ has a $k$ th harmonic derivative almost everywhere in $E$.

LeMnua. Under the condition of Theorem 1 or condition (a) of Theorem $2 f$ is locally bounded near a.e. $x_{0} \in E$.

Pro of. Let $F_{j}=\left\{x \in E:|f(x)|<j\right.$ and $\mid \sum_{i=1}^{N} A_{i} f\left(x+\varrho \sigma\left(v_{i}\right)|+B f(x)|<j \varrho^{k}\right.$ for $0<\varrho<1 / j\}$. Apply the desymmetrization lemma to $F_{j}$. For a.e. $x_{0} \in F_{j},\left|x-x_{0}\right|<\delta_{x_{0}}<1 / j$, there is at $r, 0<r<\left|x-x_{0}\right|$, and $\sigma \in \mathbb{S O}(n)$ with the property that $x-r \sigma\left(v_{1}\right) \in F_{j}$ and $x-r \sigma\left(v_{1}\right)+r \sigma\left(v_{i}\right) \in F_{j}$, $i=2, \ldots, N$.

$$
\begin{aligned}
&\left|A_{1} f(x)\right| \leqslant\left|\sum_{i=1}^{N} A_{i} f\left(x-r \sigma\left(v_{1}\right)+r \sigma\left(v_{i}\right)\right)+B f\left(x-r \sigma\left(v_{1}\right)\right)\right|+ \\
&+\left|\sum_{i=2}^{N} A_{i} f\left(x-r \sigma\left(v_{1}\right)+r \sigma\left(v_{i}\right)\right)+B f\left(x-r \sigma\left(v_{1}\right)\right)\right| \\
& \leqslant j r^{k}+j\left(\sum_{i=2}^{N}\left|A_{i}\right|+|B|\right) .
\end{aligned}
$$


We may restrict $f$ and $E$ and assume that $f$ is bounded and has compact support.

We set $u(x, y)=P_{y} * f(x)$ - the Poisson integral of $f$.

We will use the following theorems about harmonic functions $u(x, y)$ defined in the upper half space $\boldsymbol{R}_{+}^{n+1}$ [9]. We fix $\alpha>0$ and $h>0$. $\Gamma\left(x_{0}\right)$ will denote the truncated cone, $\left\{(x, y):\left|x-x_{0}\right|<\alpha y, 0<y<h\right\}$.

THEOREM. If $u$ is non-tangentially bounded in $E$, then $u$ has a nontangential limit almost everywhere in $E$.

THEOREM. If $\frac{\partial^{k} u}{\partial y^{k}}$ has non-tangential limits in $E$, then the same is true almost everywhere in $E$ for $P(\partial / \partial x) u$ when $P(x)$ is a homogeneous polynomial in $y, x_{1}, \ldots, x_{n}$ of degree $k$.

THEOREM. For any integral $k>0$, in order that $u$ have non-tangential limits almost everywhere in $E$, it is necessary and sufficient that a generalized area integral

$$
A_{k}\left(x_{0}\right)=\iint_{\Gamma\left(x_{0}\right)} y^{2 k-n-1}\left|\frac{\partial^{k} u}{\partial y^{k}}(x, y)\right|^{2} d x d y
$$

be finite for a.e. $x_{0} \in E$.

THEOREM. If $Q_{1}(x), \ldots, Q_{d}(x)$ are homogeneous polynomials of degree $r$ and the common complex zeroes satisfy $x_{1}^{2}+\ldots+x_{n}^{2}=0$, then

(a) The non-tangential boundedness of $Q_{i}(\partial / \partial x) u, i=1, \ldots, d$, in a set $E$ imply the existence of non-tangential limits a.e. of $P(\partial / \partial x) u$ when $P$ is any homogeneous polynomial of degree $r$ in $y, x_{1}, \ldots, x_{n}$.

(b) The finiteness of $\iint_{\Gamma\left(x_{0}\right)} y^{2 k-n-1}\left|Q_{i}(\partial / \partial x) u(x, y)\right|^{2} d x d y, i=1, \ldots, d$, in a set $E$ imply the finiteness a.e. of $\iint_{\Gamma\left(x_{0}\right)} y^{2 k-n-1}|P(\partial \mid \partial x) u|^{2} d x d y$ when $P$ is any homogeneous polynomial in $y, x_{1}, \ldots, x_{n}$ of degree $r$. In particular, if $a=\left(\alpha_{1}, \ldots, \alpha_{n}\right)$ is any non-negative $n$-tuple with $|a|=\sum_{i=1}^{n} \alpha_{i}<r$, then the finiteness of

$$
\iint_{\Gamma\left(x_{0}\right)} y^{2(r-|\alpha|)-n-1}\left|Q_{i}(\partial \mid \partial x) u(x, y)\right|^{2} d x d y, \quad i=1, \ldots, d,
$$

implies the finiteness of

$$
\iint_{\Gamma\left(x_{0}\right)} y^{2(r-|\alpha|)-n-1}\left|\frac{\partial^{r}}{\partial x^{\alpha} \partial y^{r-|\alpha|}} u(x, y)\right|^{2} d x d y
$$

for almost every $x_{0} \in E$. This in turn implies the existence a.e. of non-tangential limits of $\frac{\partial^{|a|}}{\partial x^{a}} u(x, y)$ by the preceding theorem.
$N$ Let $Q$ be a homogencous polynomial of smallest degree for which $\sum_{i=1}^{N} A_{i} Q\left(v_{i}\right) \neq 0$. $Q$ may be written $Q(x)=P(x)+|x|^{2} R(x)$ where. $P$ is harmonic and $R$ is of degree $m-2$ (see [2]). If $\sum_{i=1}^{N} A_{i} P\left(v_{i}\right) \neq 0$, we take $P$ as our $Q$. Otherwise, $\sum_{i=1}^{N} A_{i}\left|v_{i}\right|^{2} R\left(v_{i}\right)=\sum_{i=1}^{N} A_{i} R\left(v_{i}\right) \neq 0$. By our definition of $m, R$ must be a constant function and $\sum_{i=1}^{N} A_{i} \neq 0$.

In this latter case we shall prove Theorem 1 by showing that $\partial^{2} u / \partial y^{2}$ is non-tangentially bounded a.e. in $E$. Theorem 2 shall be proved by showing that

$$
\iint_{\Gamma\left(x_{0}\right)} y^{1-n}\left|\frac{\partial^{2} u}{\partial y^{2}}(x, y)\right|^{2} d x d y
$$

is finite almost everywhere in $t$. The preceding theorems may be applied to show that $u$ has a second or first harmonic derivative respectively when these quantities are finite. The estimates on $\partial^{2} u / \partial y^{2}$ used in obtaining these results are identical to those shown below in the case of $Q(x)$ a harmonic polynomial. The algebra is considerably easier and will not be shown.

Let $Y_{1}(x), \ldots, Y_{d}(x)$ be an orthonormal basis for the homogeneous harmonic polynomials of degree $m$. To prove Theorem 1 we shall show that $Y_{i}(\partial / \partial x) u(x, y)$ is n.t. bounded a.e. in $E$. To prove Theorem 2 we shall show that

$$
\iint_{\Gamma\left(x_{0}\right)} y^{2(m-k)-n-1}\left|Y_{i}(\partial / \partial x) u(x, y)\right|^{2} d x d y
$$

is finite a.e. in $E$. The following lemma shows that the $Y_{i}$ satisfy the conditions for the $Q_{i}$ of the preceding theorem.

LEMMA. The only common zero of $Y_{1}, \ldots, Y_{d}$ is $x=0$.

Proof. For $1 \leqslant j, k \leqslant n, j \neq k,\left(x_{j}+i x_{k}\right)^{m}$ and $\left(x_{j}-i x_{k}\right)^{m}$ are homogeneous harmonic polynomials of degree $m$. They are simultaneously zero only when $x_{j}=x_{k}=0$.

In the following lemma $P_{y}(x)=\frac{c_{n} y}{\left(|x|^{2}+y^{2}\right)^{\frac{1}{(n+1)}}}$ is the Poisson kernel.

LEMma. Let $Q$ be a homogeneous harmonic polynomial of degree $\mathrm{m}$. Then

$$
Q(\partial / \partial x) P_{y}(x)=C_{m} y Q(x)\left(|x|^{2}+y^{2}\right)^{-1(n+2 m+1)} ;
$$

$C_{0}=c_{n}$ and $C_{m}=-(n+2 m-1) C_{m-1}$.

Proof. When $m$ is zero the lemma is obvious. Consider now $Q$ of the form $Q(x)=\left(x_{1}+i x_{2}\right)^{m}$. Assume that the lemma holds for polynomials 
of degree $m-1$. We write $|x|^{2}=\left(x_{1}+i x_{2}\right)\left(x_{1}-i x_{2}\right)+x_{3}^{2}+\ldots+x_{n}^{2}$. Since

$$
\left(\frac{\partial}{\partial x_{1}}+i \frac{\partial}{\partial x_{2}}\right)\left(x_{1}+i x_{2}\right)=0 \text { and }\left(\frac{\partial}{\partial x_{1}}+i \frac{\partial}{\partial x_{2}}\right)\left(x_{1}-i x_{2}\right)=2 \text {, }
$$

we have

$$
\begin{aligned}
& \left(\frac{\partial}{\partial x_{1}}+i \frac{\partial}{\partial x_{2}}\right)^{m} P_{y}(x)=\left(\frac{\partial}{\partial x_{1}}+i \frac{\partial}{\partial x_{2}}\right) \times \\
& \times\left[C_{m-1} y\left(x_{1}+i x_{2}\right)^{m-1}\left(\left(x_{1}+i x_{2}\right)\left(x_{1}-i x_{2}\right)+x_{3}^{2}+\ldots+x_{n}^{2}+y^{2}\right)^{-\frac{1}{2}(n+2 m-1)}\right] \\
& \quad=-2 \cdot\left(\frac{n+2 m-1}{2}\right) C_{m-1} y\left(x_{1}+i x_{2}\right)^{m}\left(|x|^{2}+y^{2}\right)^{-\frac{1}{3}(n+2 m+1)} .
\end{aligned}
$$

Hence, the lemma holds for $Q$ of the form $Q(x)=\left(x_{1}+i x_{2}\right)^{m}$. We may change coordinates by replacing $x$ by $\sigma^{-1}(x)$ for any $\sigma \in \mathrm{SO}(n)$. The lemma thus holds for a polynomial $P(x)=Q\left(\sigma^{-1}(x)\right)$. Since the space of homogeneous harmonic polynomials of degree $m$ with real coefficients is an irreducible representation space for $\mathrm{SO}(n)$ under the action $\left(R_{\sigma} P\right)(x)$ $=P\left(\sigma^{-1}(x)\right)$, the image of $R_{\sigma}$ (the real part of $\left.Q\right)$ under $\mathrm{SO}(n)$ spans this space so the lemma holds for all homogeneous harmonic polynomials.

We regard the vector space over $\boldsymbol{R}$ spanned by $Y_{1}, \ldots, Y_{d}$ as a representation space for an irreducible unitary representation of $\mathrm{SO}(n)$ (see [2]). We denote by $Z^{v}(x)$ the zonal harmonic of degree $m$ with respect to the direction $v$. Then $Z^{v}(\sigma(x))=Z^{v}(x)$ whenever $\sigma(v)=v$. We choose $Y_{1}(x)=Z^{N}(x)$ where $N$ is the unit vector $(1,0, \ldots, 0)$. The entries $Y_{1}(\sigma(N)), \ldots, Y_{d}(\sigma(N))$ occupy the first column of the representation matrix for $\sigma$ with respect to this basis. Hence

$$
\begin{aligned}
\sum_{j=1}^{d} Y_{j}(\tau(N)) Y_{j}(\sigma \tau(N)) & =\left(R_{\tau^{-1}} R_{(\sigma \tau)}\right)_{11}=\left(R_{\tau^{-1} \sigma \tau}\right)_{11}=Y_{1}\left(\tau^{-1} \sigma \tau(N)\right) \\
& =Z^{\tau(N)}(\sigma \tau(N)):
\end{aligned}
$$

We may use the preceding lemma and the fact that

$$
\int_{\boldsymbol{R}^{n}} Q(\partial / \partial x) P_{y}(x) d x=Q(0) \text { for any polynomial } Q
$$

to write

$$
\begin{aligned}
A_{i} & Y_{j}(\partial \mid \partial x) u\left(x_{0} y\right) \\
& =\int_{\boldsymbol{R}^{n}} \frac{C_{m} y Y_{j}(t)}{\left(|t|^{2}+y^{2}\right)^{\frac{1}{(n+2 m+1)}}}\left(A_{i} f\left(x_{0}+t\right)-A_{i} f\left(x_{0}\right)\right) d t \\
& =\int_{0}^{\infty} \int_{\text {So }(n)} \frac{C_{m} y \varrho^{m} Y_{j}(\sigma(v))}{\left(\varrho^{2}+y^{2}\right)^{\frac{1}{2}(n+2 m+1)}}\left(A_{i} f\left(x_{0}+\varrho \sigma(v)\right)-A_{i} f\left(x_{0}\right)\right) d \sigma \varrho^{n-1} d \varrho .
\end{aligned}
$$

We let $\tau_{i}$ be any rotation for which $\tau_{i}(N)=v_{i}, i=1, \ldots, N$.

$$
\begin{aligned}
& A_{i} \sum_{j=1}^{d} Y_{j}\left(\tau_{i}(N)\right) Y_{j}(\partial / \partial x) u\left(x_{0}, y\right) \\
= & \int_{0}^{\infty} \int_{\text {so }(n)} \frac{C_{m} y \varrho^{m} \sum_{j=1}^{d} Y_{j}\left(\tau_{i}(N)\right) Y_{j}\left(\sigma \tau_{i}(N)\right)}{\left(\varrho^{2}+y^{2}\right)^{\frac{1}{2}(n+2 m+1)}}\left(A_{i}\left(f\left(x_{0}+\varrho \sigma \tau_{i}(N)\right)-f\left(x_{0}\right)\right)\right) d \sigma \varrho^{n-1} d \varrho \\
= & \int_{0}^{\infty} \int_{\operatorname{so}(n)} \frac{C_{m} y \varrho^{m} Z^{v_{i}}\left(\sigma\left(v_{i}\right)\right)}{\left(\varrho^{2}+y^{2}\right)^{\frac{1}{(n+2 m+1)}}}\left(A_{i} f\left(x_{0}+\varrho \sigma\left(v_{i}\right)\right)-A_{i} f\left(x_{0}\right)\right) d \sigma \varrho^{n-1} d \varrho .
\end{aligned}
$$

We would like to use our control of $f$ by adding up these integrals for $i=1, \ldots, N$. However, when $n>2, Z^{v_{k}}\left(\sigma\left(v_{k}\right)\right)$ does not generally equal $Z^{v_{j}}\left(\sigma\left(v_{j}\right)\right)$.

Recall from the representation theory that, as a function of $\sigma$, $f\left(x_{0}+\varrho \sigma\left(v_{i}\right)\right)$ is a linear combination of the $Y_{j}\left(\tau_{i}^{-1} \sigma \tau_{i}(N)\right)$ plus some function which is orthogonal to the entry functions of this representation. Also, the entry functions themselves are pairwise orthogonal. Let $\chi_{m}(\sigma)$ denote the character of the representation. With respect to the basis where $Y_{j}\left(\tau_{i}^{-1} \sigma \tau_{i}(N)\right), j=1, \ldots, d$, occupy the first column,

$$
\chi_{m}(\sigma)=\sum_{j=1}^{d} R_{j j}(\sigma)=Z^{v_{i}}\left(\sigma\left(v_{i}\right)\right)+\sum_{j=2}^{d} R_{j j}(\sigma) .
$$

By, orthogonality,

$$
\int_{\operatorname{so}(n)} Z^{v_{i}}\left(\sigma\left(v_{i}\right)\right) f\left(x_{0}+\varrho \sigma\left(v_{i}\right)\right) d \sigma=\int_{\operatorname{so}(n)} \chi_{m}(\sigma) f\left(x_{0}+\varrho \sigma\left(v_{i}\right)\right) d \sigma
$$

Now we can add and obtain

(3) $\quad \sum_{i=1}^{N} A_{i} \sum_{j=1}^{a} Y_{j}\left(v_{i}\right) Y_{j}(\partial / \partial x) u\left(x_{0}, y\right)$

$$
=\int_{0}^{\infty} \int_{\mathrm{sO}(n)} \frac{C_{m} y \varrho^{m} \chi_{m}(\sigma)}{\left(\varrho^{2}+y^{2}\right)^{\frac{1}{2}(n+2 m+1)}}\left(\sum_{i=1}^{N} A_{i} f\left(x_{0}+\varrho \sigma\left(v_{i}\right)\right)+B f\left(x_{0}\right)\right) d \sigma \varrho^{n-1} d \varrho .
$$

If $\sum_{i=1}^{N} A_{i} f\left(x_{0}+\varrho \sigma\left(v_{i}\right)\right)+B f\left(x_{0}\right)=O\left(\varrho^{m}\right)$, the hypothesis of Theorem 1 , then $(6)$ is dominated by $C \int_{0}^{\infty} y(\varrho+y)^{-n-1} \varrho^{n-1} d \varrho$ which is uniformly bounded as $y$ tends to zero. 
Now suppose that $f$ satisfies condition (b) of Theorem 2. We have the estimates

$y \varrho^{m}\left(\varrho^{2}+y^{2}\right)^{-\frac{1}{2}(n+2 m+1)} \leqslant A y^{-n-m} \quad$ and $\quad y \varrho^{m}\left(\varrho^{2}+y^{2}\right)^{-\frac{1}{2}(n+2 m+1)} \leqslant A^{\prime} y \varrho^{-n-m-1}$.

We set $\left|\Delta_{e}\right|=\int_{\text {So(n) }}\left|\sum_{i=1}^{N} A_{i} f\left(x_{0}+\varrho \sigma\left(v_{i}\right)\right)+B f\left(x_{0}\right)\right| d \sigma$. We obtain

$$
\begin{aligned}
\left|\sum_{i=1}^{N} A_{i} \sum_{j=1}^{d} Y_{j}\left(v_{i}\right) Y_{j}(\partial \mid \partial x) u\left(x_{0}, y\right)\right| & \\
& \leqslant A y^{-n-m} \int_{\varrho \leqslant y}\left|\Delta_{\varrho}\right| \varrho^{n-1} d \varrho+A^{\prime} y \int_{\varrho>y}\left|\Delta_{\varrho}\right| \varrho^{-n-m-1} \varrho^{n-1} d \varrho \\
& =I_{1}(y)+I_{2}(y) .
\end{aligned}
$$

By Schwarz's inequality

$$
\begin{aligned}
&\left|I_{1}(y)\right|^{2} \leqslant A^{2} y^{-2 n-2 m}\left(\int_{\varrho \leqslant y}\left|\Delta_{\varrho}\right|^{2} \varrho^{-(n+1)} \varrho^{n-1} d \varrho\right)\left(\int_{\varrho \leqslant y} \varrho^{(n+1)} \varrho^{n-1} d \varrho\right) \\
&=B y^{1-2 m} \int_{\varrho \leqslant y}\left|\Delta_{e}\right|^{2} \varrho^{-2} d \varrho \\
& \int_{0}^{\infty} y^{2(m-k)-1}\left|I_{1}(y)\right|^{2} d y \leqslant B \int_{0}^{\infty} y^{-2 k} \int_{\varrho \leqslant y}\left|\Delta_{\varrho}\right|^{2} \varrho^{-2} d \varrho \\
&=B \int_{0}^{\infty}\left|\Delta_{\varrho}\right|^{2} \varrho^{-2}\left(\int_{\varrho}^{\infty} y^{-2 k} d y\right) d \varrho=B^{\prime} \int_{0}^{\infty}\left|\Delta_{\varrho}\right|^{2} \varrho^{-2 k-1} d \varrho<\infty
\end{aligned}
$$

Similarly,

$$
\begin{gathered}
\left|I_{2}(y)\right|^{2} \leqslant\left(A^{\prime}\right)^{2} y^{2}\left(\int_{\varrho>y}\left|\Delta_{\varrho}\right|^{2} \varrho^{-2 n-2 m-2} \varrho^{n+2} \varrho^{n-1} d \varrho\right)\left(\int_{\varrho>y} \varrho^{-n-2} \varrho^{n-1} d \varrho\right) \\
=B \int_{\varrho>y}\left|\Delta_{\varrho}\right|^{2} \varrho^{-2 m-1} d \varrho \\
\int_{0}^{\infty} y^{2(m-k)-1}\left|I_{2}(y)\right|^{2} d y \leqslant B \int_{\varrho>0}\left|\Delta_{\varrho}\right|^{2} \varrho^{-2 m-1}\left(\int_{0}^{\varrho} y^{2(m-k-1)} d y\right) d \varrho \\
=B^{\prime} \int_{\varrho>0}\left|\Delta_{\varrho}\right|^{2} \varrho^{-2 k-1} d \varrho<\infty .
\end{gathered}
$$

This gives us radial control of the expression

$$
\sum_{i=1}^{N} A_{i} \sum_{j=1}^{d} Y_{j}\left(v_{i}\right) Y_{j}(\partial / \partial x) u\left(x_{0} y\right) \quad \text { for } \quad x_{0} \in E .
$$

Observe, in fact, that our estimates hold uniformly in $\mathrm{SO}(n)$ if we replace the $v_{i}$ by $\sigma\left(v_{i}\right)$.
Recall the homogeneous harmonic polynomial $P$ which had the property that $\sum_{i=1}^{N} A_{i} P\left(v_{i}\right) \neq 0 . P$ is a linear combination of the $Y_{j}$. Hence, we know that $\sum_{i=1}^{N} A_{i} Y_{j}\left(v_{i}\right) \neq 0$ for some $j$. The representation of $\operatorname{SO}(n)$ acts on $\boldsymbol{R}^{d}$ by sending the non-zero vector $\left(\sum_{i=1}^{N} A_{i} Y_{1}\left(v_{i}\right), \ldots, \sum_{i=1}^{N} A_{i} Y_{d}\left(v_{i}\right)\right)$ into the vector $\left(\sum_{i=1}^{N} A_{i} Y_{1}\left(\sigma^{-1}\left(v_{i}\right)\right), \ldots, \sum_{i=1}^{N} A_{i} Y_{d}\left(\sigma^{-1}\left(v_{i}\right)\right)\right)$. Since the representation is irreducible, the image of this vector under the action of $\mathrm{SO}(n)$ spans $\boldsymbol{R}^{d}$. Thus, there are real numbers $B_{h l}, 1 \leqslant h, l<d$, and $\sigma_{l}$ with the property that

$$
\sum_{l=1}^{a} B_{h l} \sum_{i=1}^{N} A_{i} Y_{j}\left(\sigma_{l}\left(v_{i}\right)\right)=\delta_{h j}
$$

So

$$
Y_{h}(\partial / \partial x) u\left(x_{0}, y\right)=\sum_{i=1}^{d} B_{h l}\left(\sum_{i=1}^{N} A_{i} \sum_{j=1}^{d} Y_{j}\left(\sigma_{l}\left(v_{i}\right)\right) Y_{j}(\partial / \partial x) u\left(x_{0}, y\right)\right) .
$$

Using our estimates for the inner summand, we obtain the result that $Y_{h}(\partial / \partial x) u\left(x_{0}, y\right)$ is uniformly bounded as $y \rightarrow 0$ and

$$
\int_{0}^{\infty} y^{2(m-k)-1}\left|Y_{h}(\partial / \partial x) u\left(x_{0}, y\right)\right|^{2} d y<\infty \quad \text { for } \quad x_{0} \in E
$$

under the conditions of Theorems 1 and 2, respectively.

We now proceed to obtain non-tangential control. As before, we observe that

$$
\begin{aligned}
& A_{i} \sum_{j=1}^{d} Y_{j}\left(v_{i}\right) Y_{j}(\partial / \partial x) u\left(x_{0}+r v_{i}, y\right) \\
&= \int_{0}^{\infty} \int_{\text {So }(n)} \frac{\sigma_{m} y \sum_{j=1}^{d} Y_{j}\left(\tau_{i}(N)\right) Y_{j}\left(r v_{i}-\varrho \sigma \tau_{i}(N)\right)}{\left(\left|r v_{i}-\varrho \sigma \tau_{i}(N)\right|^{2}+y^{2}\right)^{z(n+2 m+1)}} A_{i}\left(f\left(x_{0}+\varrho \sigma\left(v_{i}\right)\right)-f\left(x_{0}\right)\right) d \sigma^{n-1} d \varrho \\
&= \int_{0}^{\infty} \int_{S O(n)} \frac{C_{m} y Y_{1}\left(r N-\varrho \tau_{i}^{-1} \sigma \tau_{i}(N)\right)}{\left(r^{2}+\varrho^{2}-2 r \varrho \tau_{i}^{-1} \sigma \tau_{i}(N) \cdot N+y^{2}\right)^{\frac{1}{(n+2 m+1)}}} \times \\
& \times A_{i}\left(f\left(x_{0}+\varrho \sigma\left(v_{i}\right)\right)-f\left(x_{0}\right)\right) d \sigma \varrho^{n-1} d \varrho .
\end{aligned}
$$

As before, if $\mathrm{SO}(n)$ were abelian, we could add these expressions for $i=1, \ldots, N$. When $n>2$ this cannot be done. Set

$$
P(r, \varrho, y, \sigma)=\frac{C_{m} y Y_{1}(r N-\varrho \sigma(N))}{\left(r^{2}+\varrho^{2}-2 r \varrho \sigma(N) \cdot N+y^{2}\right)^{\frac{1}{(n+2 m+1)}}} .
$$


We seek a function $\tilde{P}(r, \varrho, y, \sigma)$ with the property that

$\int_{\mathrm{so}(n)} \tilde{P}(r, \varrho, y, \sigma) f\left(x_{0}+\varrho \sigma \tau_{i}(N)\right) d \sigma=\int_{\mathrm{SO}(n)} P\left(r, \varrho, y, \tau_{i}^{-1} \sigma \tau_{i}\right) f\left(x_{0}+\varrho \sigma \tau_{i}(N)\right) d \sigma$.

That is, $\tilde{P}$ should have the same effect as $P$ as a convolution kernel operating on functions defined on the sphere. It is clear that $\tilde{P}$ should be a central function in $\sigma$. That is, $\tilde{P}\left(r, \varrho, y, \tau^{-1} \sigma \tau\right)$ should equal $P(r, \varrho, y, \sigma)$. $\tilde{P}$ in effect, masks the non-commutativity of $\mathrm{SO}(n)$. We should also require that $\tilde{P}$ satisfy the non-tangential estimate,

$$
|\tilde{P}(r, \varrho, y, \sigma)| \leqslant A_{a} y(\varrho+y)^{-n-m-1} \quad \text { for } \quad r<\alpha y, \alpha>0 .
$$

Observe that $P$ satisfies such an estimate. When $r=0$ we saw that $\tilde{P}$ could be constructed by replacing a zonal harmonic by a character. When $r \neq 0$ the construction of $\tilde{P}$ follows this idea but requires more work. We construct $\tilde{P}$ in Section II. Here we assume the existence of $\tilde{P}$.

(4) $\quad \sum_{i=1}^{N} A_{i} \sum_{j=1}^{d} Y_{j}\left(v_{i}\right) Y_{j}(\partial / \partial x) u\left(x_{0}+r v_{i}, y\right)$

$$
=\int_{0}^{\infty} \int_{\operatorname{so}(n)} \tilde{P}(r, \varrho, y, \sigma)\left(\sum_{i=1}^{N} A_{i} f\left(x_{0}+\varrho \sigma\left(v_{i}\right)\right)+B f\left(x_{0}\right)\right) \varrho^{n-1} d \sigma d \varrho .
$$

Using the estimate for $\tilde{P}$ and proceeding exactly as in the radial case, we obtain that if $f$ satisfies the condition of Theorem 1 , then $(4)$ is uniformly bounded for $r<\alpha y$ as $y$ tends to zero. Similarly, under condition (b) of Theorem 2

$$
\int_{y>a^{-1} r} y^{2(m-k)-1}\left|\sum_{i=1}^{N} A_{i} \sum_{j=1}^{d} Y_{j}\left(v_{i}\right) Y_{j}(\partial / \partial x) u\left(x_{0}+r v_{i}, y\right)\right|^{2} d y<\infty .
$$

We shall show that we have similar control over $Y_{h}(\partial / \partial x) u\left(x_{0}+v, y\right.$, for $|v|<\alpha y$ and $h=1, \ldots, d$.

The vectors $\left(Y_{1}\left(\sigma\left(v_{1}\right)\right), \ldots, \boldsymbol{Y}_{d}^{\prime}\left(\sigma\left(v_{1}\right)\right)\right)$ span $\boldsymbol{R}^{d}$ as $\sigma$ runs through $\mathrm{SO}(n)$. We can find a $K>0$ with the property that the measure of the set of $\left(\sigma_{1}, \ldots, \sigma_{d}\right) \in \mathrm{SO}(n)^{d}$ with the property

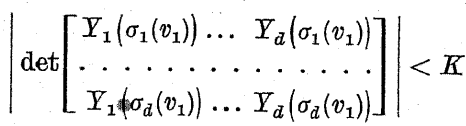

$$
\text { is smaller than }\left(n \frac{1}{2} \int_{\mathrm{So}(n)} d \sigma \varrho^{n-1} d \varrho\right)^{d}=(n c)^{d} \text {. }
$$

Let $S$ denote the complement of this set in $\operatorname{SO}(n)^{d}$.

Suppose that $f$ satisfies the condition of Theorem 1 . Let $F_{M}$ be the set of $x \in E$ for which $\left|Y_{j}(\partial / \partial x) u(x, y)\right|<M$ for $y>0$ and $j=1, \ldots, d$ and $\left|\sum_{i=1}^{N} A_{i} \sum_{j=1}^{d} Y_{j}\left(\sigma\left(v_{i}\right)\right) Y_{j}(\partial / \partial x) u\left(x+r \sigma\left(v_{i}\right), y\right)\right|<M$, for $r<\alpha y$. We apply the corollary to the desymmetrization lemma to $F_{M}$ with $\varepsilon=1$. For a.e. $x \in F_{M}$ we can found a $\delta_{x}>0$ such that if $|v-x|<\delta_{x}$ there is an $r<|v-x|$ with the property that

$$
\int_{\mathrm{so}(n)} \chi_{E}\left(x-r \sigma\left(v_{1}\right)\right) \prod_{i=2}^{N} \chi_{E}\left(x-r \sigma\left(v_{1}\right)+r \sigma\left(v_{i}\right)\right) d \sigma \geqslant n c .
$$

By the preceding remarks we know that we can find $\left(\sigma_{1}, \ldots, \sigma_{d}\right) \in S$ with the property that $v-r \sigma_{l}\left(v_{1}\right) \in F_{M}$ and $v-r \sigma_{l}\left(v_{1}\right)+r \sigma_{l}\left(v_{i}\right) \in F_{M}$, $l=1, \ldots, d, i=2, \ldots, N$. We can find a bounded set of real numbers $B_{h l}(v)$ with a bound depending only on $K$ such that

$$
A_{1} \sum_{l=1}^{l} B_{h l}(v) Y_{j}\left(\sigma_{l}\left(v_{1}\right)\right)=\delta_{h j} ;
$$

$Y_{h}(\partial / \partial x) u(v, y)=A_{1} \sum_{l=1}^{d} B_{h l}(v) \sum_{j=1}^{d} Y_{j}\left(\sigma_{l}\left(v_{1}\right)\right) Y_{j}(\partial / \partial x) u\left(v-r \sigma_{l}\left(v_{1}\right)+r \sigma_{l}\left(v_{i}\right), y\right)$.

Hence, for $|v-x|<\alpha y<\delta_{x}$,

(5) $\left|\sum_{i=1}^{N} A_{i} \sum_{l=1}^{d} B_{h l}(v) \sum_{j=1}^{d} Y_{j}\left(\sigma_{l}\left(v_{1}\right)\right) Y_{j}(\partial / \partial x) u\left(v-r \sigma_{l}\left(v_{1}\right)+r \sigma_{l}\left(v_{i}\right), y\right)\right|<O M$.

The constant depends only on $K$ and is independent of $v$. The terms for $i=2, \ldots, N$ are uniformly bounded since $v-r \sigma_{l}\left(v_{1}\right)+r \sigma_{l}\left(v_{i}\right) \in F_{M}$. Thus, $\left|Y_{h}(\partial \mid \partial x) u(v, y)\right|<C^{\prime} M$ for $|v-x|<\alpha y$ and $Y_{h}(\partial \mid \partial x) u$ has non-tangential limits a.e. in $F_{M}$ and hence almost everywhere in $E$. We have seen that this implies that all mixed partial derivatives of order $m$ have non-tangential limits a.e. in $E$. So $f$ has a $k$ th harmonic derivative in $E$ and therefore $k$ th Peano derivatives.

Now suppose that $f$ satisfies the conditions of Theorem 2. Let $F_{M}$ be the set of $x \in E$ such that

(1) $\int_{0}^{\infty} y^{2(m-k)-1}\left|Y_{i}(\partial / \partial x) u(x, y)\right|^{2} d y<M, i=1, \ldots, d$, and

(2) $\int_{y>a^{-1_{r}}} y^{2(m-k)-1} \mid \sum_{i=1}^{N} A_{i} \sum_{j=1}^{d} Y_{j}\left(\sigma\left(v_{i}\right)\left|Y_{j}(\partial \mid \partial x) u\left(x+r \sigma\left(v_{i}\right), y\right)\right|^{2} d y<M\right.$ and

(3) $x$ satisfies the result of the desymmetrization lemma with $\delta_{x}=1 / M$. $F_{M}$ is measurable and $E=\bigcup_{M=1}^{\infty} F_{M}$. For $v$ satisfying $|v-x|<1 / M$ and $x \in F_{M}$ we choose $\sigma_{1}, \ldots, \sigma_{d}$ and $B_{h l}(v)$ as above. Arguing as above, we obtain

(6)

$\int_{1 / M>y>|v-x| \mid \alpha} y^{2(m-k)-1}\left|Y_{h}(\partial \mid \partial x) u(v, y)\right|^{2} d y<C M, \quad h=1, \ldots, d$. 
We wish to show that for a.e. $x \in F_{M}$ and $\Gamma(x)=\{(v, y):|v-x|$ $<\alpha y<1 / M\}$

$$
\iint_{\Gamma(x)} y^{2(m-k)-1-n}\left|Y_{h}(\partial / \partial x) u(v, y)\right|^{2} d v d y<\infty
$$

It will suffice to show that

$$
\int_{F_{M}} \int_{\Gamma(x)} y^{2(m-k)-1-\eta}\left|Y_{h}(\partial \mid \partial x) u(v, y)\right|^{2} d v d y d x<\infty .
$$

Let $\psi(x, v, y)$ be the characteristic function of $\{(x, v, y):|v-x|<\alpha y<1 / M\}$

Rewrite (7) as

$$
\int_{F_{M}} \psi(x, v, y) d x \leqslant m\{x:|v-x|<\alpha y\}=C y^{n} .
$$

$$
\begin{aligned}
\int_{\boldsymbol{R}^{n}} \int_{0}^{\infty}\left(\int_{F_{M}} \psi(x, v, y) d x\right) y^{2(m-k)-1-n}\left|Y_{h}(\partial / \partial x) u(v, y)\right|^{2} d y d v \\
<B \int_{(v, y) \in} \int_{\substack{\mathcal{U}_{F_{M}} \\
\int_{M}}} y^{2(m-k)-1}\left|Y_{h}(\partial / \partial x) u(v, x)\right|^{2} d y d v .
\end{aligned}
$$

Since $F_{M}$ is bounded, the measure of $\left\{v\right.$ : distance $\left.\left(v, F_{M}\right)<1 / M\right\}$ is finite. By (6), the above integral is also finite. The theory of the area integral tells us that

$$
\iint_{\Gamma(x)} y^{2(m-k)-1-n}\left|\frac{\partial^{m}}{\partial x^{\alpha} \partial y^{m-k}} u(v, y)\right|^{2} d v d y<\infty
$$

for almost every $x \in E, \alpha$ any non-negative $n$-tuple of order $k$. This in turn implies that $f$ has a kth harmonic derivative in $E$ and hence, as we have seen above, a kth Peano derivative almost everywhere in $E$.

II. A central version of the Poisson kernel. It remains to find a function $\tilde{P}(r, \varrho, y, \sigma)$ which is a central function of $\sigma$ and for which

$$
\begin{aligned}
& \int_{\mathrm{SO}(n)} \tilde{P}(r, \varrho, y, \sigma) f(\sigma \tau(N)) d \sigma \\
& =\int_{\mathrm{SO}(n)} \frac{C_{m} y Z_{m}^{N}\left(r N-\varrho \tau^{-1} \sigma \tau(N)\right) f(\sigma \tau(N))}{\left(r^{2}+\varrho^{2}-2 r \varrho \tau^{-1} \sigma \tau(N) \cdot N+y^{2}\right)^{\frac{1}{2}(n+2 m+1)}} d \sigma .
\end{aligned}
$$

We also require that $\tilde{P}(r, \varrho, \sigma) \leqslant A_{\alpha} y(\varrho+y)^{-n-m-1}$ for $r<a y$.

We shall first construct $\tilde{P}$ for $m=0$, that is, for the Poisson kernel itself. Now if $Z_{m}^{N}=a_{m} x_{1}^{m}+a_{m-2} x_{1}^{m-2}|x|^{2}+\ldots$, then

$$
\begin{aligned}
& \frac{C_{m} y Z_{m}^{N}(r N-\varrho \sigma(N))}{\left(r^{2}+\varrho^{2}-2 r \varrho \sigma(N) \cdot N+y^{2}\right)^{ \pm(n+2 m+1)}} \\
= & \left(a_{m} \frac{\partial^{m}}{\partial r^{m}}-a_{m-2} \frac{\partial^{m}}{\partial r^{m-2} \partial y^{2}}+a_{m-4} \frac{\partial^{m}}{\partial r^{m-4} \partial y^{4}}-\cdots\right) \frac{c_{n} y}{\left(|r N-\varrho \sigma(N)|^{2}+y^{2}\right)^{\mathbf{z}(n+1)}} .
\end{aligned}
$$

The identities $x_{1}=r N$ and $\frac{\partial^{2}}{\partial y^{2}} P_{y}(x)=-\sum_{i=1}^{n} \frac{\partial^{2}}{\partial x_{i}^{2}} P_{y}(x)$ were used in the above equality. Once we find $\tilde{P}$ for $m=0$ we may take these derivatives (and prove estimates) to construct the proper version for any $m$.

We write the Poisson kernel as

$$
\frac{c_{n} y}{(2 \varrho r)^{\frac{1}{2}(n+1)}\left(\left(\varrho^{2}+r^{2}+y^{2}\right) / 2 \varrho r-\sigma(N) \cdot N\right)^{\frac{1}{(2(n+1)}}} .
$$

Set $a=\left(\varrho^{2}+r^{2}+y^{2}\right) / 2 \varrho r$.

Lemina. For $a>0, r<a y$ implies $a \geqslant 1+\delta_{a}$ for some $\delta_{a}>0$.

Proof. If $\varrho \leqslant 2 r$,

If $\varrho>2 r$,

$$
a=\frac{\varrho^{2}+r^{2}}{2 \varrho r}+\frac{y^{2}}{2 \varrho r} \geqslant 1+\frac{1}{4 a^{2}} .
$$

$$
a>\frac{5}{4}+\frac{y^{2}}{2 \varrho r}>\frac{5}{4} .
$$

Let $s=a-\sqrt{a^{2}-1}$. This solves $a=\left(1+s^{2}\right) / 2 s$. For $a \geqslant 1+\delta$, $0 \leqslant s \leqslant 1-\delta^{\prime}$. Recall ([12], p. 145) that the Poisson kernel for the sphere is

$$
\begin{aligned}
P(s, \sigma(N)) & =\frac{1-s^{2}}{\left(s^{2}-2 s \sigma(N) \cdot N+1\right)^{n / 2}}=\frac{1-s^{2}}{(2 s)^{n / 2}\left(\left(1+s^{2}\right) / 2 s-\sigma(N) \cdot N\right)^{n / 2}} \\
& \left.=\sum_{k=0}^{\infty} d_{k} s Z_{k}^{N} \mid \sigma(N)\right) .
\end{aligned}
$$

The dimension of the space of homogeneous harmonic polynomials of degree $k, d_{k}$, is of the order $k^{n-2}$.

We shall first find a central analog of

$$
(a-\sigma(N) \cdot N)^{-n / 2}=\frac{(2 s)^{n / 2}}{1-s^{2}} \sum_{k=0}^{\infty} d_{k} s^{k} Z_{k}^{N}(\sigma(N)) .
$$

A fractional integration in $a$ will produce an analog of $(a-\sigma(N) \cdot N)^{-\frac{1}{(2(n+1)}}$. Fubini's theorem justifies the interchange of the fractional integration and the convolution in (8).

We have seen earlier that

$$
\int_{S O(n)} Z_{l_{k}}^{N}\left(\tau^{-1} \sigma \tau(N)\right) f(\sigma \tau(N)) d \sigma=\int_{\mathrm{SO}(n)} \chi_{k}(\sigma) f(\sigma \tau(N)) d \sigma
$$

This suggests that we define $(a-\overparen{\sigma(N)} \cdot N)^{-n / 2}$ by

$$
(a-\overline{\sigma(N)} \cdot N)^{-n / 2}=\frac{(2 s)^{n / 2}}{1-s^{2}} \sum_{k=0}^{\infty} d_{k} s^{k} \chi_{k}(\sigma) .
$$


The series is dominated by the geometric series $\sum_{k=0}^{\infty} k^{2(n-2)} s^{k}$ which converges uniformly for $0 \leqslant s \leqslant 1-\delta^{\prime}$. The derivatives of (9) with respect to $s$ (or a) also converge uniformly. For large $a, s=a-\sqrt{a^{2}-1} \sim 1 / 2 a$. We may find central functions $b_{k}(\sigma)$ for which

$$
(a-\widetilde{\sigma(N)} \cdot N)^{-n / 2}=\sum_{k=0}^{\infty} b_{k}(\sigma) a^{-k-n / 2} .
$$

The serjes and its derivatives converge uniformly for $a>1+\delta$.

$$
\begin{aligned}
(a-\widetilde{\sigma(N)} \cdot N)^{-\frac{1}{(2(n+1)}} & =C \frac{d^{1 / 2}}{d a^{1 / 2}}\left(\sum_{k=0}^{\infty} b_{k}(\sigma) a^{-k-n / 2}\right) \\
& =c \frac{d}{d a} \int_{a}^{\infty}\left(\sum_{k=0}^{\infty} b_{k}(\sigma) t^{-k-n / 2}\right)(t-a)^{-1 / 2} d t \\
& =\sum_{k=0}^{\infty} b_{k}^{\prime}(\sigma) a^{-k-\frac{1}{k}(n+1)} \\
& =a^{-\frac{1}{(2(n+1)}} \sum_{k=0}^{\infty} b_{k}^{\prime}(\sigma) a^{-k} .
\end{aligned}
$$

We set

$$
\begin{aligned}
\tilde{P}(r, \varrho, y, \sigma) & =\frac{e_{n} y}{(2 \varrho r)^{ \pm(n+1)}} a^{-\frac{z}{(n+1)}} \sum_{k=0}^{\infty} b_{k}^{\prime}(\sigma) a^{-k} \\
& =\frac{c_{n} y}{\left(\varrho^{2}+r^{2}+y^{2}\right)^{\xi^{(n+1)}}} \sum_{k=0}^{\infty} b_{k}^{\prime}(\sigma) a^{-k}
\end{aligned}
$$

We have seen that $\tilde{P}$ satisfies (8) for $m=0$. We shall now show that

$$
\frac{\partial^{m}}{\partial y^{2 j} \partial r^{m-2 j}} \tilde{P}(r, \varrho, y, \sigma)<A_{a} y(\varrho+y)^{-n-m-1}
$$

for $r<a y$.

\section{LEMMa. We have}

$$
\frac{\partial^{m}}{\partial y^{2 j} \partial r^{m-2 j}} \tilde{P}=C y\left(\varrho^{2}+r^{2}+y^{2}\right)^{-\frac{1}{(2(n+2 m+1)}} \sum_{k=0}^{\infty} Q_{k}^{(m, j)}(r, \varrho, y, \sigma) a^{-k}
$$

where $Q_{k}^{(m, j)}(r, \varrho, y, \sigma)$ is a homogeneous polynomial in $r, \varrho$, and $y$ of degree $m$ whose coefficients are central functions of $\sigma$.
Proof. It is true for $m=0$. Assume that the lemma is true for $m-1$, $\frac{\partial^{m-1}}{\partial y^{2 j} \partial r^{m-1-2 j}} \tilde{P}=c y\left(\varrho^{2}+r^{2}+y^{2}\right)^{-\frac{1}{2}(n+2 m-1)} \sum_{k=0}^{\infty} Q_{k}^{(m-1, j)}(r, \varrho, y, \sigma) a^{-k}$.

A simple computation using the identities $(2 \varrho r) a=\varrho^{2}+r^{2}+y^{2}$ and $\partial a / \partial r=2(r-a \varrho)(2 \varrho r)^{-1}$ produces the result

$$
\frac{\partial^{m}}{\partial y^{2 j} \partial r^{m-2 j}} \tilde{P}=c y\left(\varrho^{2}+r^{2}+y^{2}\right)^{-\frac{1}{(}(n+2 m+1)} \sum_{k=0}^{\infty} Q_{k}^{(m, j)} a^{-k},
$$

where

$$
\begin{aligned}
Q_{k}^{(m, j)}=-(n+2 m-1) r Q_{k}^{(m-1, j)}+\left(\varrho^{2}+r^{2}+y^{2}\right) \frac{\partial Q_{k}^{(m-1, j)}}{\partial r}- \\
\quad-2 k r Q_{k}^{(m-1, j)}+2(k+1) \varrho Q_{k+1}^{(m-1, j)} .
\end{aligned}
$$

A similar computation using the identity $\partial a / \partial y=2 y(2 \varrho r)^{-1}$ produces a similar expression for $\frac{\partial^{m+1}}{\partial y^{2(j+1)} \partial r^{m-1-2 j}} \tilde{P}$. When we differentiate with respect to $y$ an odd number of times the result does not contain the factor $y$.

Since the differentiated series converges uniformly and absolutely for $a>1+\delta_{a}$, we obtain

$$
\begin{aligned}
& \frac{\partial^{m}}{\partial y^{2 j} \partial r^{m-2 j}} \tilde{P}(r, \varrho, y, \sigma) \\
& \leqslant B y(\varrho+r+y)^{-n-m-1}\left(1+A_{a} a^{-1}\right) \\
& \leqslant A_{a}^{\prime} y(\varrho+y)^{-n-m-1} \quad \text { for } \quad r<a y . \\
& \text { Adding up the terms for }\left(a_{m} \frac{\partial^{m}}{\partial r^{m}}-a_{m-2} \frac{\partial^{m}}{\partial r^{m-2} \partial y^{2}}+a_{m-4} \frac{\partial^{m}}{\partial r^{m-4} \partial y^{4}}\right) \tilde{P},
\end{aligned}
$$
we obtain the desired function and the desired estimate for any $m$.

\section{References}

[1] A. P. Calderón and A. Zyg mund, Local properties of solutions of elliptic partial differential equations, Studia Math. 20 (1961), pp. 171-225.

[3] R. R. Coifm an and G. Weiss, Representations of compact groups and spherical harmonics, L'Ens. Math. 14 (1968), pp. 121-173.

[3] A. Khintehine, Recherches sur la structure des fonctions measurables, Fund. Math. 9 (1923), pp. 212-279.

[4] J. Marcinkiewicz, Sur les series de Fourier, ibid. 27 (1936), pp. 38-69.

[5] - Sur quelques integrales din type de Dini, Ann. Soc. Pol. Math. 17 (1938), pp. 42-50.

[6] - Sur quelques integrales du la dérivée seconde généralisée, Bull. Sém. Math. Univ. Wilno 2 (1939), pp. 35-40.

[7] S. Saks, Theory of the integral, Warszawa 1937, Chapter IX. 
[8] E. M. Stein, Lecture notes, spring 1966, unpublished.

[9] - On the theory of harmonic functions of several variables, II, Acta Math. 106 (1961), pp. 137-174.

[10] - Singular integrals and differentiability properties of functions, Princeton 1970 .

[11] - Singular integrals, harmonic functions and differentiability properties of functions of several variables, Proc. Symp. Pure Math. 10 (1967), pp. 316-335.

[12] E. M. Stein and G. Weiss, Introduction to Fourier analysis on Euclidean spaces, Chapter IV, Princeton 1971.

[13] E. M. Stein and A. Zygmund, Oh the differentiability of functions, Studia Math. 23 (1964), pp. 247-283. 